\title{
Oxidation of KCNB1 channels in the human brain and in mouse model of Alzheimer's disease
}

\author{
Yu Wei ${ }^{1}$, Mi Ryung Shin ${ }^{1}$ and Federico Sesti (i)
}

\begin{abstract}
Oxidative modification of the voltage-gated $\mathrm{K}^{+}$channel subfamily B member 1 (KCNB1, Kv2.1) is emerging as a mechanism of neuronal vulnerability potentially capable of affecting multiple conditions associated with oxidative stress, from normal aging to neurodegenerative disease. In this study we report that oxidation of KCNB1 channels is exacerbated in the post mortem brains of Alzheimer's disease (AD) donors compared to age-matched controls. In addition, phosphorylation of Focal Adhesion kinases (FAK) and Src tyrosine kinases, two key signaling steps that follow KCNB1 oxidation, is also strengthened in AD vs. control brains. Quadruple transgenic mice expressing a non-oxidizable form of KCNB1 in the 3XTg-AD background (APP ${ }_{S W E}, P S 1_{M 146 V}$, and tau ${ }_{P 301 L}$ ), exhibit improved working memory along with reduced brain inflammation, protein carbonylation and intraneuronal $\beta$-amyloid $(A \beta)$ compared to 3xTg-AD mice or mice expressing the wild type (WT) KCNB1 channel. We conclude that oxidation of KCNB1 channels is a mechanism of neuronal vulnerability that is pervasive in the vertebrate brain.
\end{abstract}

\section{Introduction}

The imbalance between the production of reactive oxygen species (ROS) and the ability of the cells to detoxify them, referred to as oxidative stress, is a hallmark of aging and a number of pathologies ${ }^{1}$. One protein known to undergo oxidation in the brain is the voltagegated $\mathrm{K}^{+}$channel subfamily B member $1(\mathrm{KCNB} 1)^{2}$. ROS induce cross-linking of KCNB1 subunits to each other (oligomerization) via the formation of disulfide bridges. Once oxidized, KCNB1 channels do not conduct current and accumulate in the plasma membrane from where they activate an outside-in signaling pathway mediated by integrins-with whom they form macromolecular complexes-focal adhesion kinases (FAK), Src tyrosine kinases and c-Jun $\mathrm{N}$-terminal (JNK) kinases ${ }^{3,4}$. The concerted action of these kinases stimulates the production of more

\footnotetext{
Correspondence: Federico Sesti (federico.sesti@rutgers.edu)

${ }^{1}$ Department of Neuroscience and Cell Biology, Rutgers University Robert

Wood Johnson Medical School, 683 Hoes Lane West, Piscataway, NJ 08854, USA

Edited by A. Verkhrtasky
}

ROS and induces apoptosis. Traumatic brain injury (TBI) has provided a good model to assess the consequences of KCNB1 oxidation in vivo, because oxidative stress is extensive in this condition $^{5,6}$. Transgenic mice overexpressing a non-oligomerizable variant of human KCNB1 (Tg-C73A) exhibit reduced tissue damage and improved behavioral outcome following TBI compared to non-Tg mice or transgenic mice expressing the wild type (WT) KCNB1 channel. Moreover, those effects can be neutralized by Dasatinib, a Src inhibitor, which directly impinges the downstream effectors of oxidized KCNB1 channels, the Src tyrosine kinases ${ }^{7}$. Given the significant presence/role of oxidative stress in multiple disease states, it is likely KCNB1 oxidation may be present in conditions beyond TBI.

One such case is Alzheimer's disease (AD), a dementia characterized by multiple etiologies and pathogenic mechanisms. AD brains exhibit strong evidence of ROSmediated injury including abnormal levels of protein oxidation, DNA oxidation and lipid peroxidation ${ }^{8}$. Indeed, the oxidative stress hypothesis in Alzheimer's disease 
Table 1 Neuropathology reports of the AD and age-matched control donors that provided the post mortem hippocampal tissue used in this study

\begin{tabular}{|c|c|c|c|c|}
\hline Donor \# & Diagnosis & Age & Sex & Neuropathology report \\
\hline 1 & Control & 83 & $\mathrm{~F}$ & $\begin{array}{l}\text { 1. Neurofibrillary degeneration, Braak and Braak early stage II. } \\
\text { 2. Small defect in optic tract with loss of myelinated fibers. } \\
\text { 3. Arteriolosclerosis and atherosclerosis. } \\
\text { 4. Mild, autolysis. }\end{array}$ \\
\hline 2 & Control & 84 & M & $\begin{array}{l}\text { 1. Neurofibrillary degeneration, Braak and Braak early stage I, with rare non-neuritic neocortical amyloid } \\
\text { plaques and rare amyloid angiopathy. } \\
\text { 2. Arteriosclerosis and atherosclerosis. } \\
\text { 3. Mild Purkinje cells loss. } \\
\text { 4. Mild autolysis. }\end{array}$ \\
\hline 3 & Control & 85 & M & $\begin{array}{l}\text { 1. Neurofibrillary degeneration, late Braak stage II, with non-neuritic neocortical amyloid plaques. } \\
\text { 2. Arteriosclerosis, atherosclerosis, and a remote microhemorrhage in the substantia nigra. }\end{array}$ \\
\hline 4 & Control & 81 & M & $\begin{array}{l}\text { 1. Neurofibrillary degeneration, Braak stage II, with non-neuritic neocortical amyloid plaques. } \\
\text { 2. Arteriosclerosis and atherosclerosis. }\end{array}$ \\
\hline 5 & Control & 82 & $\mathrm{~F}$ & $\begin{array}{l}\text { 1. Neurofibrillary degeneration, Braak stage II, with sparse non-neuritic neocortical amyloid plaques. } \\
\text { 2. Small vessel cerebrovascular disease with arteriosclerosis, arteriolosclerosis, and a microinfarct in the } \\
\text { nucleus accumbens. }\end{array}$ \\
\hline 6 & Control & 80 & $\mathrm{~F}$ & $\begin{array}{l}\text { 1. Sparse non-neuritic neocortical amyloid plaques. } \\
\text { 2. Arteriosclerosis and atherosclerosis. }\end{array}$ \\
\hline 7 & Alzheimer's disease & 83 & $\mathrm{~F}$ & $\begin{array}{l}\text { 1. Alzheimer's disease, Braak stage VI, with severe amyloid angiopathy. } \\
\text { 2. Early (preclinical) Parkinson's disease (incidental Lewy body disease). } \\
\text { 3. Arteriosclerosis and atherosclerosis. }\end{array}$ \\
\hline 8 & Alzheimer's disease & 84 & M & $\begin{array}{l}\text { 1. Advanced Alzheimer's disease, Braak and Braak stage VI, with severe amyloid angiopathy. } \\
\text { 2. Small vessel cerebrovascular disease with Arteriosclerosis, arteriolosclerosis and atherosclerosis, arterial } \\
\text { mural necrosis, three microinfarcts in the neocortex, lacunes in the caudate nucleus and thalamus, and } \\
\text { focal zones of acute ischemic change in neocortex. } \\
\text { 3. Moderate autolysis. }\end{array}$ \\
\hline 9 & Alzheimer's disease & 82 & $\mathrm{~F}$ & $\begin{array}{l}\text { 1. Alzheimer's disease, Braak stage VI, with amyloid angiopathy. } \\
\text { 2. Arteriosclerosis. }\end{array}$ \\
\hline 10 & Alzheimer's disease & 82 & $\mathrm{~F}$ & $\begin{array}{l}\text { 1. Alzheimer's disease, Braak stage VI, with amyloid angiopathy and hippocampal sclerosis. } \\
\text { 2. Small vessel cerebrovascular disease with severe arteriosclerosis, arteriolosclerosis and atherosclerosis } \\
\text { and a microinfarct in the interior temporal cortex. }\end{array}$ \\
\hline 11 & Alzheimer's disease & 85 & M & $\begin{array}{l}\text { 1. Alzheimer's disease, Braak stage VI, with amyloid angiopathy and mesial temporal sclerosis (hippocampal } \\
\text { sclerosis). } \\
\text { 2. Arteriosclerosis and atherosclerosis. } \\
\text { 3. Early Parkinson's disease. }\end{array}$ \\
\hline 12 & Alzheimer's disease & 87 & M & $\begin{array}{l}\text { 1. Alzheimer's disease, Braak stage Vl, with amyloid angiopathy. } \\
\text { 2. Arteriosclerosis. }\end{array}$ \\
\hline
\end{tabular}

Donor number (controls 1-6; AD: 7-12), age in years, diagnosis, and sex are indicated

posits that ROS contribute to neurodegeneration and death through the cumulative action of multiple damaging processes. It is not coincidental that $\mathrm{KCNB} 1$ channels undergo oxidation in the 3xTg-AD triple transgenic mouse model of $\mathrm{AD}$, where their amounts increase with age $^{2,9}$. Furthermore, non-conducting KCNB1 oligomers cause enhanced calcium spike frequency and decreased Fluo- 4 intensity in primary $3 \times \mathrm{Tg}-\mathrm{AD}$ neurons ${ }^{10}$. This body of evidence underscores the potentially crucial role of oxidation of KCNB1 channels for AD but also the need 
to elucidate the impact of this mechanism on $\mathrm{AD}$ pathology.

Here, we investigate oxidation of KCNB1 channels in the post mortem human hippocampus and in mouse model of AD. The results of our studies indicate that KCNB1 channels undergo extensive oxidation in the human $\mathrm{AD}$ brain along with enhanced phosphorylation of FAK and Src kinases. In the 3xTg-AD brain, KCNB1 oxidation is associated with inflammation and oxidative stress which act in concert to increase intraneuronal $\beta$-amyloid. These cellular injures correlate with behavioral deficit,suggesting that oxidation of KCNB1 channels may contribute to human AD pathology.

\section{Results}

\section{KCNB1 undergoes oxidation in the human brain}

KCNB1 forms macromolecular complexes with integrins in the mouse brain ${ }^{4}$. These interactions are retained during the formation of KCNB1 oligomers. In fact, it is the oligomerization of KCNB1 channels that triggers integrin signaling leading to the recruitment/activation of Src tyrosine kinases via autophosphorylated FAK at Tyr397 ${ }^{4}$. To assess the mechanism of KCNB1 oxidation in the human brain we obtained post mortem hippocampal tissue of 6 AD donors ( 3 females and 3 males, average age 83.8 years) and 6 age-matched controls ( 3 females and 3 males, average age 82.5 years) from the Harvard Brain Tissue Resource Center through the Neurobiobank repository of the NIH. Donors' information including neuropathology reports is listed in Table 1 . To determine whether KCNB1 and integrins form complexes in the human brain, proteins were immunoprecipitated (IP) with an antibody that detects integrin alpha chain V (integrin$\alpha 5$ ) and immunoblotted (IB) with an antibody that detects KCNB1 or vice versa. Representative western blots (WB) of co-immunoprecipitation experiments (co-IP, $N=3$ ) in the post mortem hippocampus of $\mathrm{AD}$ donors and agematched controls are shown in Fig. 1a. Integrin- $\alpha 5$ pulled down KCNB1 channels and similarly, KCNB1 channels pulled down integrin- $\alpha 5$ suggesting that KCNB1 and integrins form stable complexes in the human hippocampus. A single KCNB1 subunit runs with a molecular mass $\sim 110 \mathrm{kDa}$. KCNB1 oligomers typically exhibit molecular masses $\sim 200 \mathrm{kDa}$ but they can range up to $\sim 400 \mathrm{kDa}^{7}$. Notably, KCNB1 oligomers were present in both normally aging and $\mathrm{AD}$ brains indicating that KCNB1 undergoes oxidation in the human brain. To quantify the extent of KCNB1 oxidation we calculated the densitometric ratio between the monomeric and oligomeric bands (oxidation ratio) that we measured from western blots of brain lysates stained with KCNB1 antibody (the oxidation ratio only depends on the relative amounts of oligomerized and non-oligomerized protein in the blot). This analysis underscored a significant $67 \%$ increase in the oxidation ratio of the AD brain vs. control (Fig. 1b). Furthermore, this increase correlated well with the increase in oxidative stress that was assessed by measuring protein carbonylation (64\% increase in $\mathrm{AD}$ vs. control, Fig. 1c). Thus, KCNB1 oxidation is a physiological process which occurs in the normally aging human brain and is exacerbated in AD, consistent with the elevated oxidative stress associated with this condition.
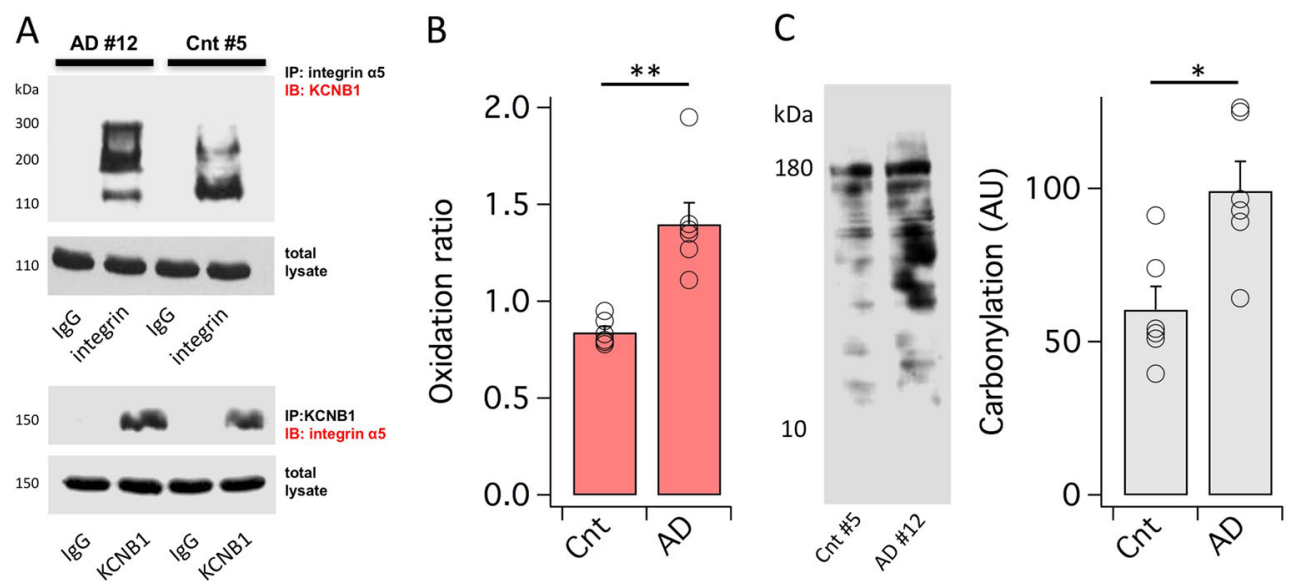

Fig. 1 KCNB1 undergoes oxidation in the human brain. a In the upper western blots, integrin- $a_{5}$ immunoprecipitates (IP) and IgG IPS (control) from AD sample \#12 and control sample \#5 and total lysates were immunoblotted (IB) with Kv2.1 primary antibody. Total lysates were treated with $2 \% \beta$-mercaptoethanol added in the sample buffer and thus do not show oligomers. Integrins pull down both single KCNB1 subunits ( $110 \mathrm{kDa})$ and oligomers ( 200 to $\sim 400 \mathrm{kDa}$ ). In the lower western blots, KCNB1 and IgG IPs and total lysates were stained with integrin- $a_{5}$ primary antibodies. b Mean oxidation ratio from six control samples and six $A D$ samples. Each point represents the average of two technical replicates. ${ }^{* *} P=0.008$. c Representative western blot of protein carbonylation (polyclonal dinitrophenyl (DNP) antibody) and mean protein carbonylation (arbitrary units) from six control and six AD samples. Each point represents the average of two technical replicates. ${ }^{*} P=0.011$ 
FAK and Src phosphorylation are increased in the AD brain

The representative western blots in Fig. 2a show total and phosphorylated FAK at Tyr397 and Src at Tyr416 in the post mortem brains of an AD donor and of an agematched control. The fraction of activated FAK and Src was increased by, respectively, $80 \%$ and $58 \%$ in $\mathrm{AD}$ vs. control brains (Fig. 2b), in good agreement with the 67\% increase in KCNB1 oxidation/oligomerization.

\section{KCNB1 is oxidized in mouse model of AD}

To further elucidate the role of KCNB1 oxidation in AD we studied it in the genetic background of the 3xTg-AD mouse model of AD. This transgenic animal expresses three dementia-related transgenes, namely $\mathrm{APP}_{\mathrm{SWE}}, \mathrm{PS}_{\mathrm{M} 146 \mathrm{~V}}$, and $\operatorname{tau}_{\mathrm{P} 301 \mathrm{~L}}$ and exhibits both plaque and tangle pathology, as well as synaptic dysfunction ${ }^{9}$. We constructed $4 x T g$ mice by cross-breeding $3 \times \mathrm{Tg}-\mathrm{AD}$ with transgenic mice that expresses a non-oligomerizabe variant of KCNB1 obtained by replacing Cys73 to Ala (Tg-C73A) or the WT channel (Tg-WT) as control, in cortex and hippocampus which we characterized previously ${ }^{7}$. We used $4 x T g$ mice heterozygous in either WT or C73A since homozygous Tg-WT mice exhibit developmental delays ${ }^{7}$. Figure $3 a$ summarizes biochemical assessment of KCNB1 oxidation in mouse model of AD. Consistent with results with post mortem human brain tissue, KCNB1 oligomerization was robust in the $3 \times \mathrm{Tg}-\mathrm{AD}$ brain and was further increased in mice overexpressing the WT channel. In contrast, KCNB1 oligomerization was low in the brains expressing the C73A variant as expected (we showed previously that exogenous and endogenous KCNB1 subunits form heteromeric complexes. The Cys to Ala mutation is dominant negative and therefore the amounts of oxidized KCNB1 channels are negligible in the $\mathrm{Tg}$-C73A brain $^{7}$ ). Furthermore, the fractions of phosphorylated FAK kinases at Tyr397 (Fig. 3b) and Src kinases at Tyr416 (Fig. 3c) were significantly increased in $4 \mathrm{xTg}-\mathrm{WT}$ and $3 \mathrm{xTg}-\mathrm{AD}$ compared to $4 \mathrm{xTg}-\mathrm{C} 73 \mathrm{~A}$ brains.

\section{KCNB1 oxidation contributes to cognitive impairment in mouse model of $A D$}

To determine the impact of KCNB1 oxidation on the cognitive function of the hippocampus, we assessed 1 -year-old mice in the spatial working memory task in the Morris Water Maze (MWM), which is one of the most well-modeled aspects of the working memory deficits of $\mathrm{AD}^{11}$. All mice behaved similarly during the acclimation trials when the platform was visible (Fig. 4a), and therefore baseline adjustments were not performed. In contrast, during the training period (Fig. 4b) and in the post-training assessment of memory consolidation with the platform removed (Fig. 4c), 4xTg-C73A mice performed significantly better than 3xTg-AD and 4xTg-WT. The superior learning abilities of $4 \mathrm{xTg}-\mathrm{C} 73 \mathrm{~A}$ mice were already apparent at the beginning of the training. During the first day, these mice learned to locate the platform twice as much as fast than $4 \mathrm{xTg}$-WT mice (gaining $\sim 14 \mathrm{~s}$ per trial vs. $\sim 7 \mathrm{~s}$ per trial of the $4 \mathrm{xTg}$-WT mice) even though all mice performed similarly in the first trial, irrespective of the genotype (Fig. $4 \mathrm{~d}$ ). Swimming speeds were comparable in the various groups of mice $(0.32 \pm$ $0.02 ; \quad 0.34 \pm 0.02$, and $0.35 \pm 0.02 \mathrm{~m} / \mathrm{s}$ for $3 \times T g-A D$, $4 \times \mathrm{Tg}-\mathrm{WT}$, and $4 \mathrm{xTg}-\mathrm{C} 73 \mathrm{~A}$, respectively), suggesting that the improved outcome in the $4 \mathrm{xTg}-\mathrm{C} 73 \mathrm{~A}$ mice was not due to increased activity and we also did not observe sexspecific differences in the various genotypes ${ }^{12}$. In previous studies we demonstrated that the only distinction between the WT channel and the C73A variant is that the latter
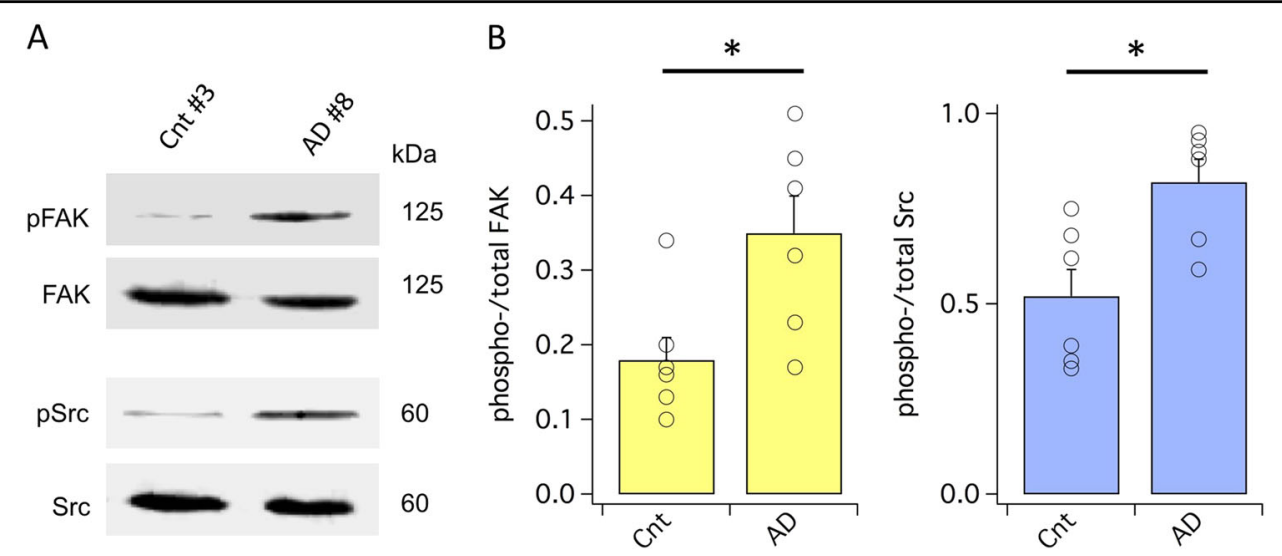

Fig. 2 FAK and Src kinases activities are increased in the human AD brain. a Representative western blots showing autophosphorylated FAK at tyr397 (pFAK) and total FAK (upper blot) and phosphorylated Src at Tyr 416 (pSrc) and total Src (lower blot) in the hippocampi of AD donor \#8 and control donor \#3. FAK protein was detected into a single, $\sim 125 \mathrm{kDa}$ band and Src protein into a single, $\sim 60 \mathrm{kDa}$ band. $\mathbf{b}$ Mean fraction of activated FAK (pFAK/FAK) and activated Src (pSrc/tSrc) from six control and six AD samples. Each point represents the average of two technical replicates. ${ }^{*} P=$ 0.028 and ${ }^{*} P=0.013$ for FAK and Src, respectively 

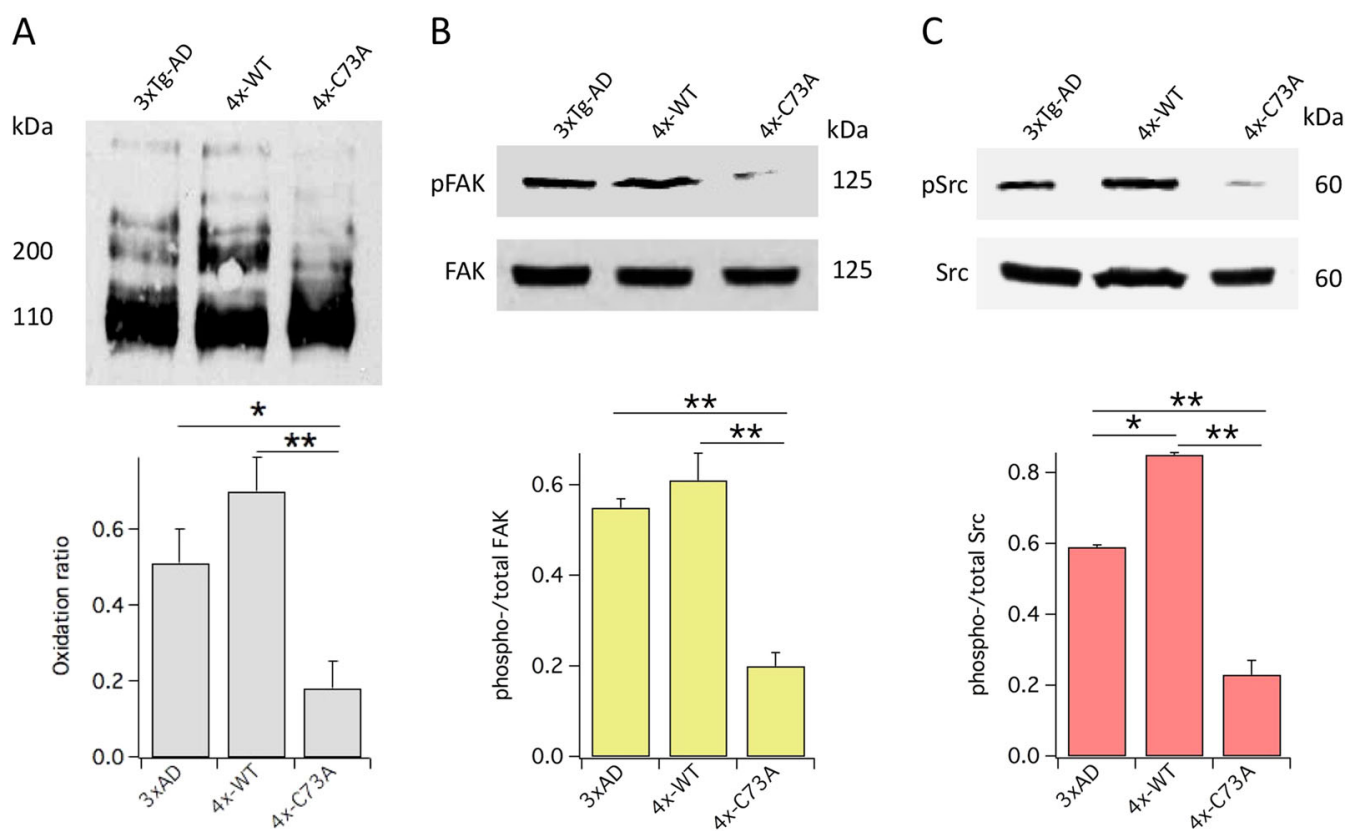

Fig. 3 KCNB1 oxidation is recapitulated in mouse model of AD. a Representative western blot showing oxidized KCNB1 channels in the brains of 6-month-old 3xTg-AD, 4xTg-WT, and 4xTg-C73A mice and mean oxidation ratio. $N=4$ brains/genotype. b Representative western blots showing autophosphorylated FAK at Tyr397 and total FAK in the brains of 6-month-old 3xTg-AD, 4xTg-WT, and 4xTg-C73A mice and mean pFAK/FAK ratio. $N=3$ brains/genotype. c Representative western blots showing phosphorylated Src at Tyr416 and total Src in the brains of 6-month-old 3xTg-AD, 4xTg-WT, and 4xTg-C73A mice and mean pSrc/Src ratio. $N=3$ brains/genotype. ${ }^{*} P<0.05$ and ${ }^{* *} P<0.01$

does not undergo oxidation in the brain. This leads us to conclude that differences between the $4 \mathrm{xTg}$ and $3 \mathrm{xTg}$ genotypes mainly depended on the oxidation of the channel and not on other factors ${ }^{4,7}$ and thus that there is a causal relationship between the extent of KCNB1 oxidation and spatial working memory in mouse model of $\mathrm{AD}$.

\section{Dasatinib ameliorates AD-like pathology in 3xTg-AD mice}

The activation of Src tyrosine kinases is a key signaling event following the formation of KCNB1 oligomers. In previous studies we identified Dasatinib, a FDA-approved specific inhibitor of Src kinases, blood-brain barrier permeable and pharmacologically active in the brain, as a means to neutralize the toxic effects of KCNB1 oxidation in mouse model of TBI (the drug is also associated with decreased KCNB1 oligomerization) $)^{7,13-23}$. Since in both TBI and AD oxidation of KCNB1 channels leads to SrC activation Dasatinib should also be effective in the latter. Two groups of 10- and 11-month-old 3xTg-AD mice were daily injected with $25 \mathrm{mg} / \mathrm{kg}$ Dasatinib, delivered intraperitoneally (ip), for 2 and 1 months respectively and then tested in the MWM. The mice treated for 1 month did not exhibit appreciable improvement compared to vehicle mice (Fig. 4e). Mice that underwent the 2 months treatment performed moderately better than vehicle mice during the 6-day training period (Fig. 4f) and in the memory consolidation test with the platform removed (Fig. 4g). Overall, the drug was less effective in 3xTg-AD mice than in TBI mice, where it could largely suppress the effects of the injury ${ }^{7}$ probably because at the time of the treatment, the $\mathrm{AD}$-like pathology was already at an advanced stage.

KCNB1 oxidation is associated to astrocytosis in the $3 \times \mathrm{Tg}$ AD brain

Astrocytosis is broad in $\mathrm{AD}$, and glial fibrillary acidic protein (GFAP) is a useful biomarker for the disease ${ }^{24-26}$. We assessed the levels of GFAP in the brain by immunohistochemistry (IHC, Fig. 5a) and western blot (Fig. 5b). Reactive astrocytes were more numerous in the hippocampi of $4 \mathrm{xTg}-\mathrm{WT}$ and 3xTg-AD compared to $4 \mathrm{xTg}-\mathrm{C} 73 \mathrm{~A}$, a result corroborated by western blot analysis. Similarly, the amounts of GFAP protein were increased in post mortem $\mathrm{AD}$ brains compared to agematched controls, as expected (Fig. 5c) ${ }^{24,25}$.

\section{Oxidation of KCNB1 contributes to inflammation and oxidative stress in the 3xTg-AD brain}

Astrocytosis generally accompanies inflammation, thus GFAP provides indirect evidence for inflammation ${ }^{27,28}$. We directly assessed inflammation by staining hippocampal sections with two antibodies (Abcam clone ab5076, Fig. 6a, c and Wako clone 019-19741, Fig. 6b, d) 

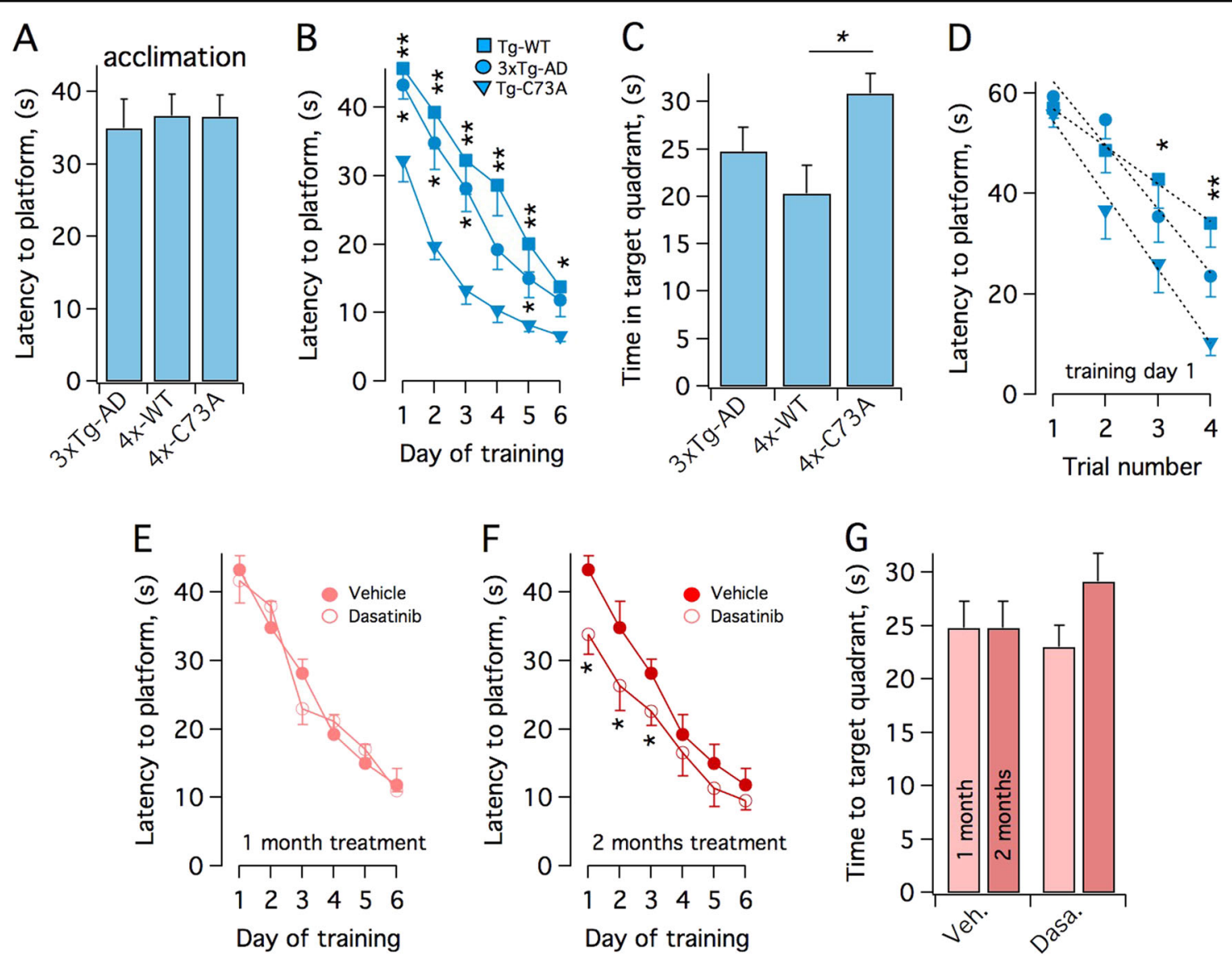

Fig. 4 Oxidation of KCNB1 causes working memory impairment in mouse model of AD a Mean latency to platform (visible) of the indicated genotypes 4 days before training. b Mean latency to platform of 3xTg-AD (circles), 4xTg-WT (squares), and 4xTg-C73A (triangles) mice. c Consolidated memory retention test (platform removed) for the indicated groups of mice. $\mathbf{d}$ Latency to platform of 3xTg-AD (circles), 4xTg-WT (squares), and 4xTg$\mathrm{C} 73 \mathrm{~A}$ (triangles) mice, in the individual trials during the first day of training. Fit of the data to a linear function (dotted lines) gave a rate of: $-14.72 \pm$ $1.09 \mathrm{~s} /$ trial for $4 \times \mathrm{Tg}-\mathrm{C} 73 \mathrm{~A} ;-11.67 \pm 1.94 \mathrm{~s} /$ trial for $3 \times \mathrm{Tg}-\mathrm{AD}$ and $-7.43 \pm 0.36 \mathrm{~s} /$ trial for $4 \times \mathrm{Tg}-\mathrm{WT}$ mice. e Mean latency to platform of 1-year-old 3xTgAD mice daily ip injected with $25 \mathrm{mg} / \mathrm{kg}$ Dasatinib (filled circles) or vehicle (hollow circles) for 1 month. Mean swimming speeds were: $0.37 \pm 0.03$ and $0.32 \pm 0.02 \mathrm{~m} / \mathrm{s}$ for vehicle and Dasatinib, respectively. $\mathbf{f}$ Mean latency to platform of 1-year-old 3xTg-AD mice daily ip injected with $25 \mathrm{mg} / \mathrm{kg}$ Dasatinib (filled circles) or vehicle (hollow circles) for 2 months. Mean swimming speeds were: $0.35 \pm 0.02$ and $0.35 \pm 0.02 \mathrm{~m} / \mathrm{s}$ for vehicle and Dasatinib, respectively. $\mathbf{g}$ Consolidated memory retention test for the indicated groups of mice. In a through $\mathbf{d} N=11$ mice/genotype. In $\mathbf{b}$ and c, statistical significance of pairwise comparisons between $4 \times \operatorname{Tg}-\mathrm{C} 73 \mathrm{~A}$ and the other genotypes is indicated. In e through (f) $N=9$ mice/genotype. ${ }^{*} P<0.05$ and ${ }^{* *} P<0.01$

against allograft inflammatory factor 1 (Iba1). Reactive microglia reactive for Iba1 were present in the hippocampus of 3xTg-AD mice (Fig. 6a, b). Reactive cells were increased in $4 x T g-W T$ sections and were significantly decreased in $4 x$ Tg-C73A sections (Fig. 6c, d). The antibodies stained accordingly to manufacturer's datasheets (clone ab5076: globular cells; clone 019-19741: branched cells); however, due to the shared lineage of microglia and macrophages, markers are common to both cell types. Therefore we cannot rule out that reactive cells are composed of both cell types, but we can reasonably conclude that inflammation is decreased in the $4 \mathrm{xTg}$-C73A brain compared to the other genotypes.

\section{Oxidative stress is low in neurons expressing $\mathrm{C73A}$}

The pathway activated in response to KCNB1 oligomerization promotes oxidative stress, a hallmark of $\mathrm{AD}^{3,7}$. Accordingly, protein carbonylation, which provides a measure of oxidative stress, was markedly lessened in the brains of 6-month-old 4xTg-C73A mice compared to 3xTg-AD and 4xTg-WT, where it was maximal (Fig. 6e).

\section{Oxidation of KCNB1 contributes to amyloidosis}

Neuroinflammation and oxidative stress sustain the synthesis of $\beta$-amyloid $(A \beta)^{29}$. The 3xTg-AD mice develops intracellular $A \beta$ in the hippocampus at around 6 months of age and plaque around 1 year'. Indeed, neurons reactive for intracellular $A \beta$ were more 

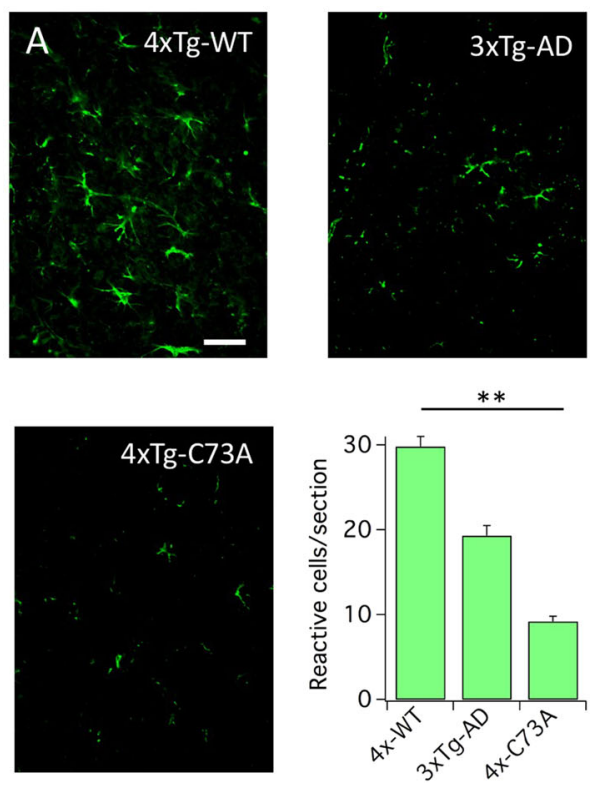

C
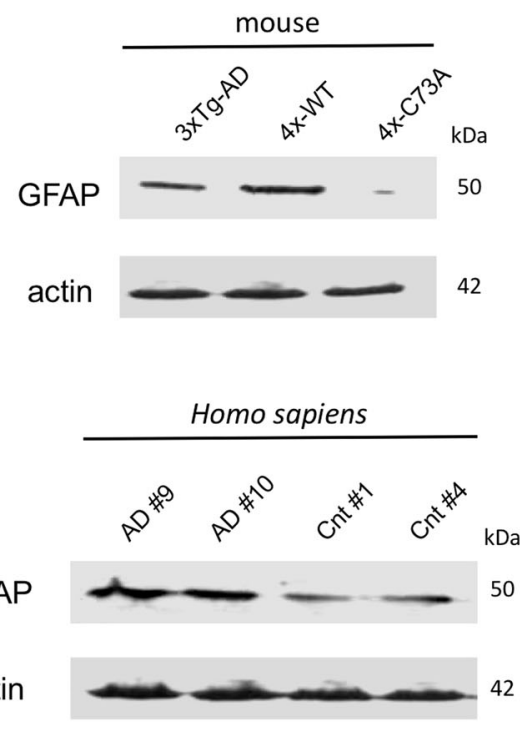

Fig. 5 KCNB1 oxidation increases astrocytosis in mouse model of AD. a Representative images of coronal hippocampal sections from the brains of the indicated genotypes stained with GFAP antibody and mean number of cells reactive for GFAP, per section. Scale bar: $200 \mu \mathrm{m}$. Each single mean was calculated from 12 sections (3 brains, 2 fields of view/section). $\mathbf{b}$ Representative western blot of GFAP protein in the brains of the indicated genotypes. c Representative western blot of GFAP protein in the brains of AD donors \#9 and \#10 and age-matched controls \#1 and \#4. ${ }^{* * P}<0.01$
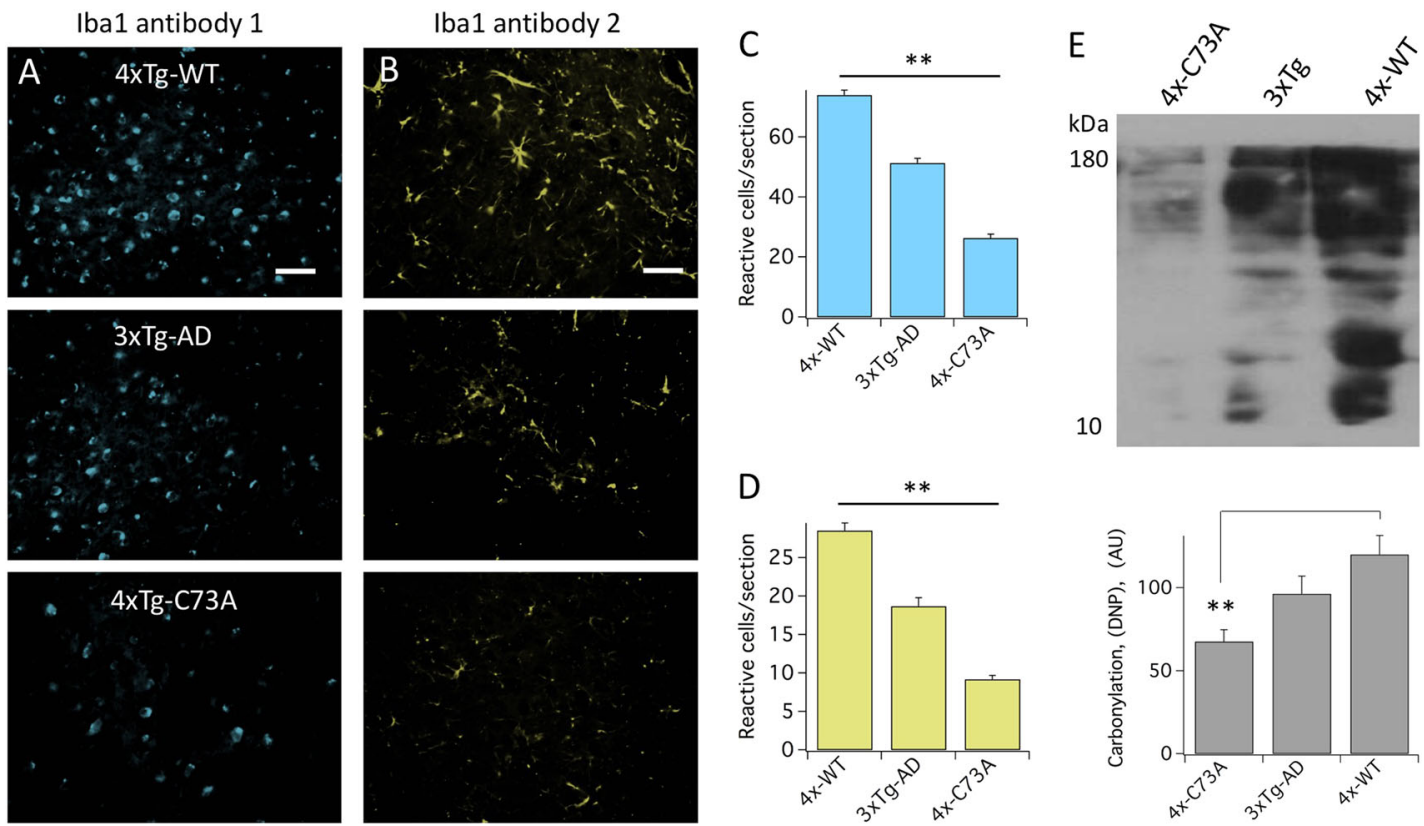

Fig. 6 KCNB1 oxidation increases inflammation in mouse model of AD. $\mathbf{a}, \mathbf{b}$ Representative images of coronal hippocampal sections from the brains of the indicated genotypes stained with an antibody against Iba1 from Abcam (a) or from Wako (b). Scale bar $100 \mu$ m. $\mathbf{c}$, d Mean number of cells reactive to Ibal Abcam (c) or Wako (d) antibody per section for the indicated genotypes. Each mean was calculated from 12 sections (3 brains, 2 fields of view/section). e Representative western blot of protein carbonylation and mean protein carbonylation (arbitrary units) in the brains of the indicated genotypes. $N=3$ brains/genotype. ${ }^{* *} P<0.01$ 

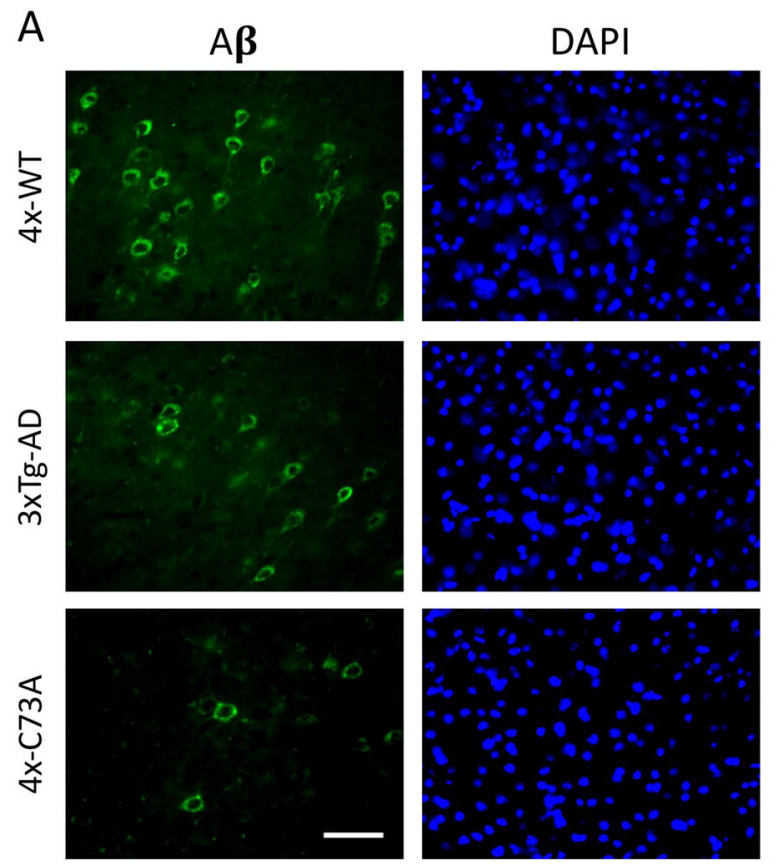
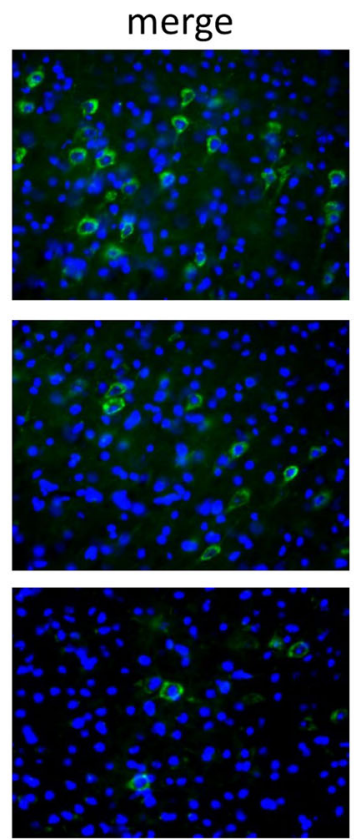

B

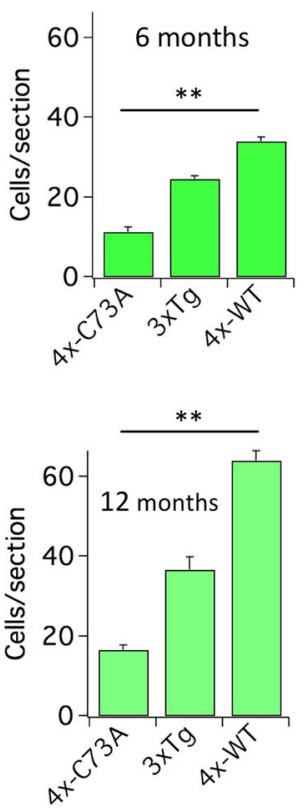

Fig. 7 KCNB1 oxidation is associated with increased intraneuronal A $\boldsymbol{\beta}$ in young mice. a Representative images of coronal hippocampal sections from the brains of the indicated 6-month-old genotypes stained with human A $\beta$ antibody and DAPI. Scale bar: $40 \mu$ m. $\mathbf{b}$ Mean number of neurons reactive for $A \beta$ per section in the hippocampi of 6-month-old (green) and 12-month-old (pale green) mice of the indicated genotypes. Each mean was calculated from 12 sections (3 brains, 2 fields of view/section)

numerous in hippocampal sections cut from the brains of 6- and 12-month-old 4xTg-WT and 3xTg-AD mice compared to $4 \mathrm{xTg}-\mathrm{C} 73 \mathrm{~A}$ mice (Fig. 7a, b). We assessed amyloid precursor protein (APP), $A \beta$ and $A \beta(1-42)$ protein by Western blot and enzyme-linked immunosorbent assay (ELISA) analysis, respectively, in the brains of 1-year-old mice. While the amounts of APP were similar in all genotypes (Fig. 8a, b) the amounts of $A \beta$ protein (Fig. 8a, c), including the most neurotoxic $A \beta(1-42)$ form (Fig. 8d), were significantly lower in the C73A brains compared to the $3 \times \mathrm{Tg}-\mathrm{AD}$ and $4 \mathrm{xTg}-\mathrm{WT}$ brains. A $\beta$ (1-42) was also moderately decreased (29\% reduction) in the brains of $3 x T g-A D$ mice treated with Dasatinib for 2 months $(3.4 \pm 0.4$ and $2.4 \pm 0.1 \mathrm{pg} / \mathrm{ml}$ in the absence/ presence of Dasatinib, $P<0.043$ one-tailed Student's $t$-test, $N=3$ brains/group, data not shown). In addition, the number of neurons reactive for $A \beta$ was increased by $88 \%$ in $4 \mathrm{xTg}$-WT brains between 6 and 12 months of age but only by $46 \%$ in $4 x$ Tg-C73A brains (Fig. 7b) suggesting that oxidation of KCNB1 exhibits an accelerating influence on the production of $A \beta$. Taken together, these data underscore a mechanistic link between oxidation of $\mathrm{KCNB} 1$ and the production of $\mathrm{A} \beta$ and further suggest that KCNB1 oligomers impact the pathogenesis of $A \beta$ early.

\section{KCNB1 oxidation contributes to tauopathy}

It has been proposed that $A \beta$ may contribute to tau hyperphosphorylation and evidence further shows that oxidative stress is interlinked with tauopathy ${ }^{30-36}$. Since oxidation of KCNB1 is associated to both amyloidosis and oxidative stress, we assessed the levels of hyperphosphorylated tau in the brains of 1-year-old mice by IHC, using an antibody that detects phosphorylated tau at Ser199 (Fig. 9a, b), and phosphorylated tau at Ser356 (Fig. 9c). The number of reactive neurons to both antibodies was maximal in 4xTg-WT hippocampi and minimal in 4xTg-C73A hippocampi. Tauopathy was less advanced than amyloidosis or inflammation, consistent with the fact that in the 3xTg-AD mouse it develops around 1 year of age, which was the maximal age reached by our animals ${ }^{9,33}$.

\section{Discussion}

Reactive oxygen species and $\mathrm{K}^{+}$channels hold key roles in both the physiology and pathology of the brain. Evidence of oxidation of $\mathrm{K}^{+}$channels in TBI as well as AD is suggestive of a general mechanism of neuronal vulnerability that can potentially affect all conditions characterized by oxidative stress, from normal aging to neuropathies ${ }^{37,38}$. To address this fundamental question we investigated oxidation of KCNB1 channels in AD, a condition associated to massive oxidative stress. Our studies reveal the presence of oxidation-induced KCNB1 oligomers in post mortem hippocampal tissue of aged human donors and, to a significantly larger extent, of AD donors. Phosphorylation of FAK and Src kinases-two key 


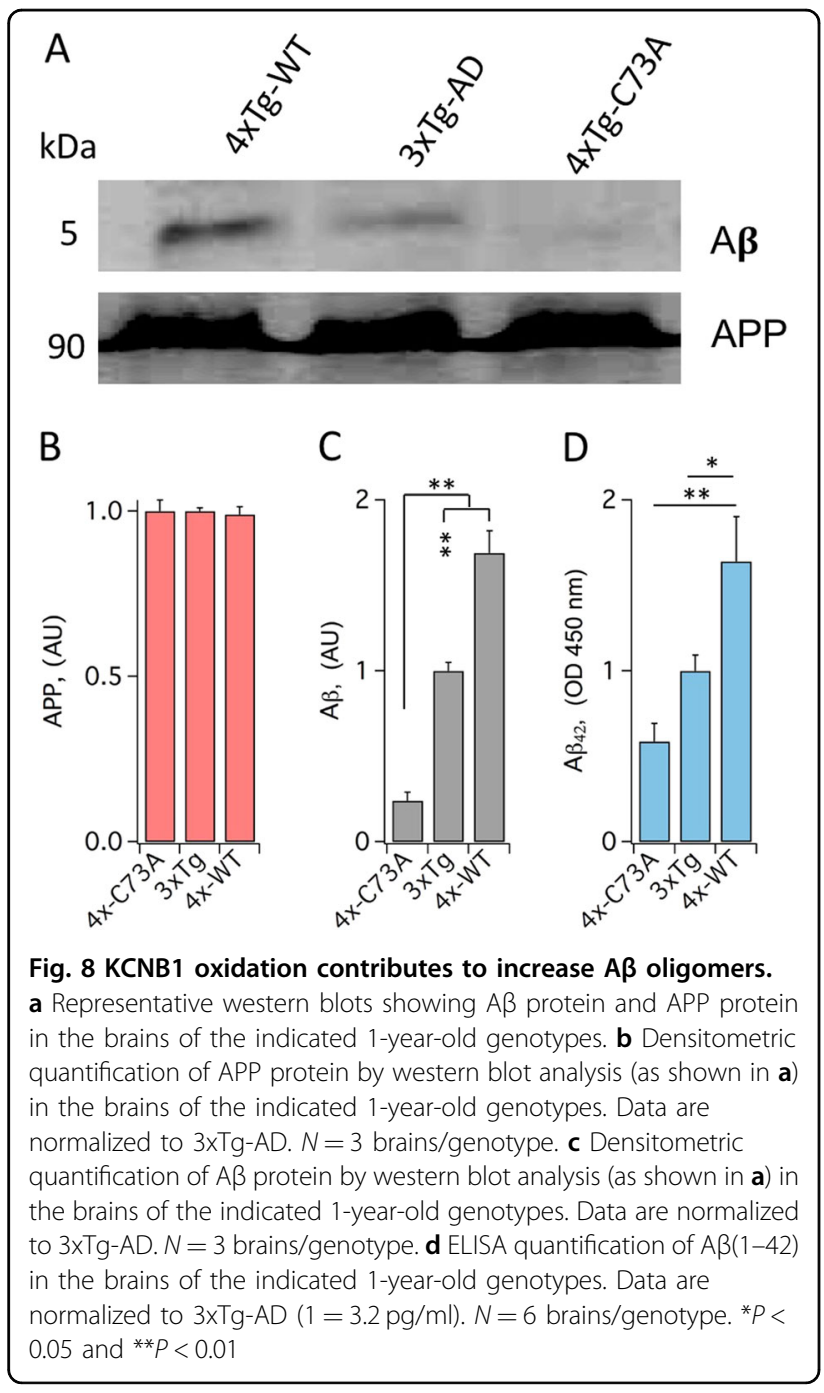

signaling events triggered by KCNB1 oxidation/oligomerization--are also markedly increased in the AD brains compared to age-matched controls. The possibility exists that KCNB1 channels had undergone oxidation during the preparation of the samples, for example, during the time elapsed between the death of the donor and the collection of the tissue. However, these technical issues should have affected the samples randomly and thus alone, cannot explain the significant differences in the extent of KCNB1 oxidation, protein carbonylation, FAK and Src phosphorylation and GFAP immunoreactivity that exist between control and AD brains. Further, oxidized KCNB1 channels are detected in the brains of normally aging mice and, in significantly larger quantities, in 3xTg-AD brains where these technical issues are minimized $^{2}$. We conclude that oxidation of KCNB1 channels is a process that takes place in the human brain under pathophysiological conditions.
To gain mechanistic insight into the role of KCNB1 oxidation in $\mathrm{AD}$, we engineered $3 \times \mathrm{Tg}$ - $\mathrm{AD}$ mice to overexpress either the C73A variant (low oxidation) or the WT channel (high oxidation) in cortex and hippocampus. Increased amounts of KCNB1 oligomers correlated with increased amounts of GFAP and Iba1, two markers for astrocytosis and inflammation, along with FAK and Src phosphorylation and protein carbonylation. At the behavioral level, 4xTg-C73A mice performed significantly better than the other genotypes in the working memory task of the MWM. KCNB1 oxidation promotes inflammation, oxidative stress, neuronal apoptosis, and behavioral deficit in mouse model of traumatic brain injury a condition that, likewise $\mathrm{AD}$, is associated to copious release of $\operatorname{ROS}^{7}$. Hence, oxidative stress contributes to neurotoxicity via modification of $\mathrm{K}^{+}$channels irrespective of the specific pathology. We conclude that oxidation of $\mathrm{K}^{+}$channels is a general mechanism of neuronal vulnerability that is conserved from worms to humans ${ }^{37}$.

Previous studies have implicated oxidized KCNB1 channels in $\mathrm{AD}$ by showing that they induce hyperexcitability in primary 3xTg-AD hippocampal neurons (oxidized KCNB1 channels do not conduct current) ${ }^{2,10}$. The work presented here adds new insight into the molecular mechanisms underlying KCNB1's neurotoxicity in $\mathrm{AD}$. Thus, neuroinflammation and oxidative stress are two well established factors that contribute to the production of $A \beta$. In turn, elevated levels of $A \beta$ can create a positive feedback loop toward generating more oxidative stress. Accordingly, in the 4xTg-WT brain, where KCNB1 oxidation is exacerbated, neuroinflammation and oxidative stress are increased and intraneuronal $A \beta$ is also increased, whereas in the $4 \mathrm{xTg}-\mathrm{C} 73 \mathrm{~A}$ brain, where KCNB1 oxidation is diminished, intraneuronal $A \beta$ is decreased along with neuroinflammation and oxidative stress. A significant reduction of the levels of intraneuronal $A \beta$ can be already observed in 6 month-old $4 \mathrm{xTg}-\mathrm{C} 73 \mathrm{~A}$ mice, an age in which plaque deposition is still absent while oxidative stress and inflammation are already elevated, suggesting that KCNB1 oxidation is an early contributor to $A \beta$ pathogenesis ${ }^{9,33}$. $A \beta$ and oxidative stress may also contribute to induce phosphorylation of tau. Indeed, we observed neurons reactive to hyperphosphorylated tau in all genotypes that following a well established pattern, were more numerous in $4 \times \mathrm{Tg}$-WT and 3xTg-AD hippocampi compared to 4xTg-C73A. However, the effects of KCNB1 oxidation on tauopathy will need further scrutiny as in the 3xTg-AD mouse this begins around 1 year of age, the maximal age reached by the mice used in this study ${ }^{9,33}$. Overall, these results are in agreement with previous studies. One of them in particular, from Rehamn and colleagues showed that inhibition of JNK kinases, which are one of the end-points of 
A
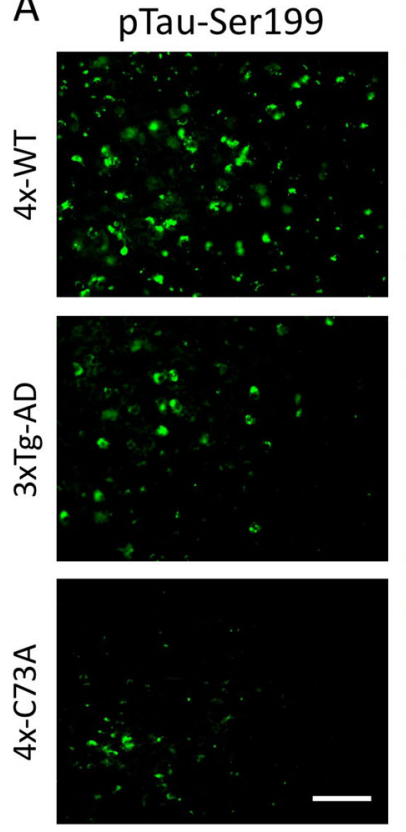

DAPI
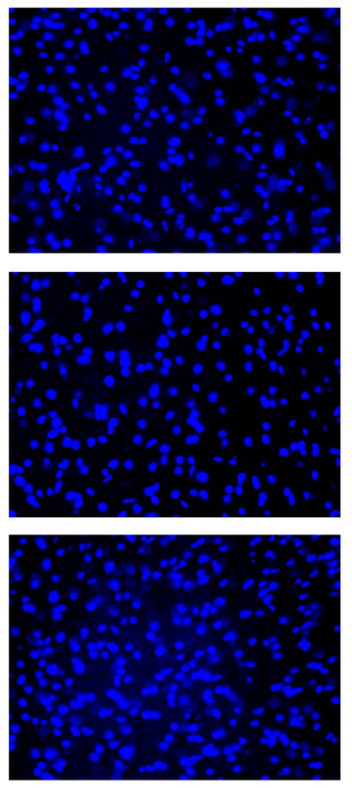

merge
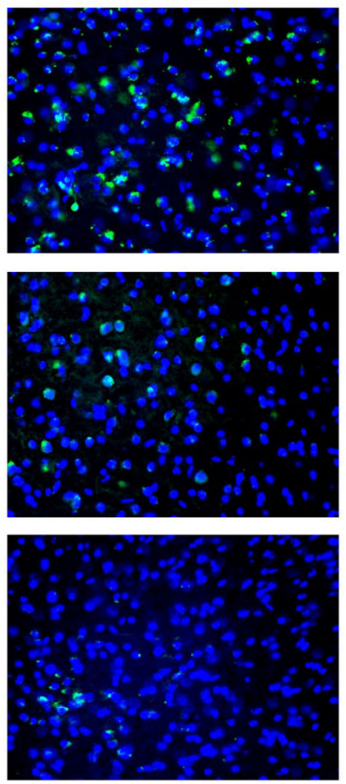

B
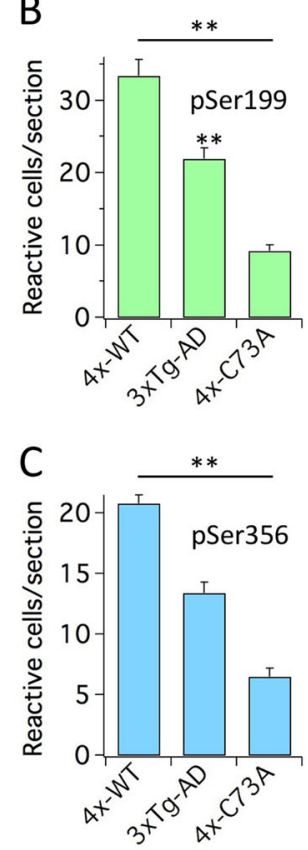

Fig. 9 KCNB1 oxidation contributes to tauopathy. a Representative images of coronal hippocampal sections from the brains of the indicated 1-year-old genotypes stained with an antibody that detects tau protein phosphorylated as Ser199 and DAPI. Scale bar: 40 Mm. b Mean number of neurons reactive for an antibody that detects tau protein phosphorylated at Ser199 per section in the hippocampi of 1-year-old mice of the indicated genotypes. Each mean was calculated from 12 sections (3 brains, 2 fields of view/section). c Mean number of neurons reactive for an antibody that detects tau protein phosphorylated at Ser356 per section in the hippocampi of 1-year-old mice of the indicated genotypes. Each mean was calculated from 12 sections (3 brains, 2 fields of view/section). ${ }^{*} P<0.01$

the signaling pathway activated by oxidized KCNB1 channels results in marked reductions of $A \beta$ production and hyperphosphorylated tau along with inflammation, apoptotic neurodegeneration and improved behavioral outcomes $^{3,39}$.

Dasatinib, which shows robust efficacy in mouse model of TBI by directly impinging on the pathway activated by KCNB1 oxidation and in the APP/PS1 mouse model of $\mathrm{AD}$, had only modest effects in preventing the $A D$-like pathology of the 3xTg-AD mouse $^{7,18}$. It is likely that oxidation of KCNB1 occurs during the early stages of the disease and thus treatments that impinge on this mechanism need to be started early. Accordingly, the drug exhibited increased efficacy in younger animals subjected to longer treatment. Dasatinib is blood-brain barrier permeable and is the first line drug for patients with Philadelphia chromosome-positive Central Nervous System (CNS) leukemia ${ }^{13-15,17}$ and CNS chronic myeloid leukemia $^{16,19,20,22,23}$. A factor that can limit Dasatinib's accumulation in the brain is $\mathrm{P}$-glycoprotein (P-gp, ABCB1), as ABCB1 knock out mice exhibit significantly higher amounts of Dasatinib in the brain compared to control $^{40-43}$. However, P-gp function decreases in the aging brain and is severely compromised in the $\mathrm{AD}$ brain $^{44-47}$. Thus, Dasatinib therapy may represent a possible strategy for the treatment of AD.

\section{Conclusions}

- This study provides the first experimental evidence that oxidative modification of KCNB1 takes place in the aging human brain and is exacerbated in the Alzheimer's brain.

- KCNB1 oxidation, cause neuroinflammation, amyloidosis, and cognitive impairment.

- Src kinase inhibitor Dasatinib may represent a possible strategy for the treatment of $\mathrm{AD}$.

\section{Materials and methods \\ Reagents}

Src (clone 2108), phospho-Src at tyr416 (clone 2101), FAK (clone 3285), and phospho-FAK at tyr397 (clone 3283) antibodies were purchased from Cell Signaling Technology (Danvers, MA). Kv2.1 antibody (clone K89/ 34) was purchased from NeuroMab, UC Davis/NIH. GFAP antibody (clone MAB3402) and actin antibody (clone MAB1501) were purchased from Millipore, Billerica, MA. Human $\beta$-amyloid antibody (clone 6E10) was purchased from Biolegend, San Diego, CA. Iba1 
antibodies were purchased from Abcam (Cambridge, MA, clone ab5076) and from Wako Chemicals (Richmond, VA, clone 019-19741). Phospho-tau antibody (Ser199) clone 2H23L4, was purchased from ThermoFisher Scientific (Waltham, MA) and phospho-tau antibody (Ser356) clone ab75603, was purchased from Abcam. Integrin- $\alpha 5$ antibody (sc-10729, now discontinued) and goat anti-rabbit IgG-R (clone sc-2091) were purchased from Santa Cruz Biotechnology (Dallas, TX). Amyloid-beta 42 ELISA kit was purchased from ThermoFisher Scientific and used following manufacturer's instructions. Protein Carbonyl Immunoblot kit was purchased from Cell Biolabs, Inc, San Diego, CA and used following manufacturer's instructions.

\section{Transgenic mice}

We obtained 4xTg-AD transgenic mice heterozygous in KCNB1 by cross-breeding 3xTg-AD mice with Tg-WT (4xTg-WT) or Tg-C73A (4xTg-C73A) mice that we previously characterized ${ }^{4,7}$. Briefly, $\mathrm{Tg}$-WT and $\mathrm{Tg}$-C73A mice overexpress human WT or C73A KCNB1 tagged to the human influenza hemagglutinin (HA) tag in the C-terminus in cortex and hippocampus driven by the thy1.2 cassette $^{48}$. These mice have been donated to the MMRRC repository of the $\mathrm{NIH}$ and are available from them (MMRRC:43829 and MMRRC:43830). In heterozygous mice, the amounts of human KCNB1 protein are comparable in the two transgenic lines and are about half the amounts of endogenous KCNB1 protein. Primary hippocampal neurons express functional KCNB1 currents, whose steady-state amplitudes at $+80 \mathrm{mV}$ are comparable in Tg-WT and Tg-C73A transgenic neurons and roughly $\sim 40 \%$ larger than in non- $\mathrm{Tg}$ neurons. The Cys73 to Ala replacement in the C73A variant does not affect channel's functional attributes nor its ability to cluster in the plasma membrane.

\section{Biochemistry}

The detailed biochemical procedure was previously described $^{4,49}$.

\section{Immunoblots}

Approximately $100 \mathrm{mg}$ of human frozen hippocampal tissue (a gift of the Harvard Brain Tissue Resource Center) or half sagittal mouse brains of either sex were homogenized with a glass tissue grinder in lysis buffer [0.32 M sucrose, $5 \mathrm{mM}$ Tris-Cl pH 6.8, $0.5 \mathrm{mM}$ EDTA, $1 \mathrm{mM}$ PMSF, and protease inhibitor cocktail set I (Calbiochem, San Diego, CA)]. Samples were centrifuged at $2000 \mathrm{rpm}$ for $10 \mathrm{~min}$ and the supernatant used for biochemical analysis. Protein content was quantified with the Bradford colorimetric assay (Sigma, St. Louis, MO) and dissolved in Laemmli buffer with or without reducing agents. Proteins were resolved by $8-12 \%$ SDS-PAGE and transferred to a
PVDF membrane that was incubated in a $5 \%$ solution of nonfat milk in Tween 20-PBS (PBST) for $2 \mathrm{~h}$ at room temperature. After overnight incubation at $4{ }^{\circ} \mathrm{C}$ with the primary antibody, the membrane was washed for $20 \mathrm{~min}$ and incubated at room temperature with the appropriate secondary antibody.

\section{Co-immunoprecipitations}

Brain lysates were incubated at $4{ }^{\circ} \mathrm{C}$ overnight in the presence of Kv2.1 or integrin- $\alpha 5$ or IgG antibodies. Then, protein A agarose beads ( $30 \mu$ l of $50 \%$ bead slurry) were added and incubated overnight at $4{ }^{\circ} \mathrm{C}$. Samples were centrifuged for $30 \mathrm{~s}$ at $4{ }^{\circ} \mathrm{C}$ and the pellet was washed five times in cell lysis buffer. The pellet was resuspended with $50 \mu \mathrm{l} 2 \times$ SDS Laemmli buffer, heated at $100^{\circ} \mathrm{C}$ for $10 \mathrm{~min}$ and centrifuged for $1 \mathrm{~min}$ at $14,000 \times g$. The samples were loaded on $8 \%$ SDS-PAGE gel and immunoblotted. The blots were washed in PBST for $20 \mathrm{~min}$ and incubated for 5 min with chemiluminescence substrates and exposed.

Densitometry analysis was performed using ImageJ (NIH) software.

\section{Immunohistochemistry}

Mice were transcardially perfused with $0.9 \%$ saline followed by $4 \%$ paraformaldehyde. The brains were extracted and cryoprotected in 30\% sucrose. Twenty micrometers frozen sections were prepared throughout the cortex and the hippocampus in a 1:20 series so that the same set of tissue samples could be used for expression of different makers. Slides were incubated overnight in with antibody at $4{ }^{\circ} \mathrm{C}$ (GFAP 1:500, all other antibodies 1;100). Slides were then incubated in the appropriate secondary antibody. All slides were mounted in VECTASHIELD Antifade Mounting Medium (Vector Laboratories, Burlingame, CA) and stored at $4{ }^{\circ} \mathrm{C}$. Staining was visualized on a Zeiss Axiophot microscope at $\times 40$. Positive were counted in coronal sections representing a 1:20 series inclusive of the entire length of the hippocampus. CA1 and CA3 as well as the dentate gyrus was used for quantitation of cells in the hippocampus.

\section{Morris water maze}

The detailed procedure for the MWM was previously described $^{7}$. Briefly, mice were acclimated to the paradigm and tested for baseline response using a visible platform test 4 days prior. The animals were placed in a circular pool of water containing non-toxic white paint and a clear platform for escape. To assess learning, mice were trained using a hidden platform fixed in one of four quadrants for 6 consecutive days ( 4 trials/day). Black and white distal cues were placed on the walls. The quadrant in which the mouse was placed was pseudo-randomly varied throughout training and the time to locate the platform was recorded. Maximum trial time was $60 \mathrm{~s}$ and the 
mouse remained or was placed on the platform for $15 \mathrm{sec}$ and warmed for $10 \mathrm{~min}$ between trials. To assess memory retention, the day after the last training session the animals were be subjected to a $60 \mathrm{~s}$ probe trial with the platform removed and the time spent in the target quadrant was measured ${ }^{50}$. Data were recorded using a video-tracking system (EthoVision XT; Noldus Information Technology, Leesburg, VA).

\section{Drug administration}

Dasatinib (LC Laboratories, Woburn, MA) was given intraperitoneally (ip) at $25 \mathrm{mg} / \mathrm{kg}$. Dasatinib was diluted in vehicle solution $(50 \mathrm{mM} \mathrm{NaAc}, \mathrm{pH}=5.0)$ from a $200 \mathrm{mg} / \mathrm{ml}$ stock in dimethyl sulfate (DMSO). Each mouse was subjected to a daily dose of either vehicle or Dasatinib solution via an ip injection.

\section{Statistical analysis}

Quantitative data are presented as mean \pm standard error of the mean (SEM). The level of significance, assumed at the $95 \%$ confidence limit or greater $(P<0.05)$, was estimated using the Student's $t$-test (http:// studentsttest.com) and one-way ANOVA with a Tukey post hoc test (http://vassarstats.net/anovalu.html).

\section{Acknowledgements}

The frozen samples of human tissue were requested through the Neurobiobank repository of the National Institutes of Health and were kindly provided by the Harvard Brain Tissue Resource Center which is supported in part by HHSN-271-2013-00030C. The 3xTg-AD mouse was a gift of Dr. Cheryl Dreyfus. We thank Dr. Surindo Singh for help with crossing the transgenic mice and Dr. Barbara Viviani for critical reading of the manuscript. This work was supported by a NSF grant (1456675) and a NIH grant (NS096619) to F.S.

Received: 26 April 2018 Revised: 23 June 2018 Accepted: 16 July 2018 Published online: 26 July 2018

\section{References}

1. Pham-Huy, L. A., He, H. \& Pham-Huy, C. Free radicals, antioxidants in disease and health. Int. J. Biomed. Sci. 4, 89-96 (2008).

2. Cotella, D. et al. Toxic role of $\mathrm{K}+$ channel oxidation in mammalian brain. J. Neurosci. 32, 4133-4144 (2012)

3. Wu, X., Hernandez-Enriquez, B., Banas, M., Xu, R. \& Sesti, F. Molecular mechanisms underlying the apoptotic effect of KCNB1 $\mathrm{K}+$ channel oxidation. J. Biol. Chem. 288, 4128-4134 (2013).

4. Yu, W., Gowda, M., Sharad, Y., Singh, S. A. \& Sesti, F. Oxidation of KCNB1 potassium channels triggers apoptotic integrin signaling in the brain. Cell Death Dis. 8, e2737 (2017).

5. Bayir, $H$. et al. Assessment of antioxidant reserves and oxidative stress in cerebrospinal fluid after severe traumatic brain injury in infants and children. Pediatr. Res. 51, 571-578 (2002)

6. Tyurin, V. A. et al. Oxidative stress following traumatic brain injury in rats: quantitation of biomarkers and detection of free radical intermediates. J. Neurochem. 75, 2178-2189 (2000).

7. Yu, W. et al. Oxidation of KCNB1 potassium channels causes neurotoxicity and cognitive impairment in a mouse model of traumatic brain injury. J. Neurosci. 36, 11084-11096 (2016).

8. Markesbery, W. R. Oxidative stress hypothesis in Alzheimer's disease. Free Radic. Biol. Med. 23, 134-147 (1997).
9. Oddo, S. et al. Triple-transgenic model of Alzheimer's disease with plaques and tangles: intracellular Abeta and synaptic dysfunction. Neuron 39, 409-421 (2003).

10. Frazzini, V. et al. Altered Kv2.1 functioning promotes increased excitability in hippocampal neurons of an Alzheimer's disease mouse model. Cell Death Dis. 7. e2100 (2016)

11. Webster, S. J., Bachstetter, A. D., Nelson, P. T., Schmitt, F. A. \& Van Eldik, L. J. Using mice to model Alzheimer's dementia: an overview of the clinical disease and the preclinical behavioral changes in 10 mouse models. Front. Genet. 5, 88 (2014).

12. Clinton, L. K. et al. Age-dependent sexual dimorphism in cognition and stress response in the 3XTg-AD mice. Neurobiol. Dis. 28, 76-82 (2007).

13. Abdelhalim, A. et al. Remission of Philadelphia chromosome-positive central nervous system leukemia after dasatinib therapy. Leuk. Lymphoma $\mathbf{4 8 ,}$ 1053-1056 (2007)

14. Porkka, K. et al. Dasatinib crosses the blood-brain barrier and is an efficient therapy for central nervous system Philadelphia chromosome-positive leukemia. Blood 112, 1005-1012 (2008).

15. Alimena, G. et al. Dasatinib in the management of lymphoid blast crisis of Philadelphia-positive chronic myeloid leukemia with multiple extra-medullary and intracranial localizations. Leuk. Res. 33, e134-e136 (2009).

16. Russwurm, G. et al. Dasatinib induces complete remission in a patient with primary cerebral involvement of B-cell chronic lymphocytic leukemia failing chemotherapy. Blood 116, 2617-2618 (2010).

17. Bhadri, V. A., Satharasinghe, K., Sugo, E., Barbaric, D. \& Trahair, T. N. Excellent response to dasatinib of childhood Philadelphia positive intracranial acute lymphoblastic leukaemia tumours. Br. J. Haematol. 152, 347-349 (2011).

18. Dhawan, G. \& Combs, C. K. Inhibition of Src kinase activity attenuates amyloid associated microgliosis in a murine model of Alzheimer's disease. J. Neuroinflamm. 9, 117 (2012).

19. Zhou, H. S. et al. Isolated central nervous system relapse in patient with blastcrisis chronic myeloid leukemia in durable complete cytogenetic remission on dasatinib treatment: pharmacokinetics and ABL mutation analysis in cerebrospinal fluid. Leuk. Lymphoma 54, 1557-1559 (2013).

20. Nishimoto, M. et al. Dasatinib maintenance therapy after allogeneic hematopoietic stem cell transplantation for an isolated central nervous system blast crisis in chronic myelogenous leukemia. Acta Haematol. 130, 111-114 (2013).

21. Oberoi, R. K., Mittapalli, R. K., Fisher, J. \& Elmquist, W. F. Sunitinib LC-MS/MS assay in mouse plasma and brain tissue: application in CNS distribution studies. Chromatographia 76, 23-24 (2013) https://doi.org/10.1007/s10337-0132528-1

22. $\mathrm{Xu}$, Z. et al. The overwhelmingly positive response to dasatinib of a patient with multiple blast crisis of chronic myeloid leukemia. Int. J. Clin. Exp. Med. 8 1460-1466 (2015)

23. Lai, S. W., Huang, T. C., Chen, J. H., Wu, Y. Y. \& Chang, P. Y. Dasatinib as the salvage therapy for chronic myeloid leukemia with blast crisis and central nervous system involvement: a case report. Oncol. Lett. 9, 1957-1961 (2015).

24. Hol, E. M. et al. Neuronal expression of GFAP in patients with Alzheimer pathology and identification of novel GFAP splice forms. Mol. Psychiatry $\mathbf{8}$, 786-796 (2003).

25. Panter, S. S. et al. Glial fibrillary acidic protein and Alzheimer's disease. Neurochem. Res. 10, 1567-1576 (1985).

26. Olsson, B. et al. CSF and blood biomarkers for the diagnosis of Alzheimer's disease: a systematic review and meta-analysis. Lancet Neurol. 15, 673-684 (2016).

27. Jacque, C. M. et al. Determination of glial fibrillary acidic protein (GFAP) in human brain tumors. J. Neurol. Sci. 35, 147-155 (1978).

28. Vos, P. E. et al. GFAP and S100B are biomarkers of traumatic brain injury: an observational cohort study. Neurology 75, 1786-1793 (2010).

29. Cai, Z., Zhao, B. \& Ratka, A. Oxidative stress and beta-amyloid protein in Alzheimer's disease. Neuromolecular. Med. 13, 223-250 (2011).

30. Zheng, W. H., Bastianetto, S., Mennicken, F., Ma, W. \& Kar, S. Amyloid beta peptide induces tau phosphorylation and loss of cholinergic neurons in rat primary septal cultures. Neuroscience 115, 201-211 (2002).

31. Lewis, J. et al. Enhanced neurofibrillary degeneration in transgenic mice expressing mutant tau and APP. Science 293, 1487-1491 (2001).

32. Gotz, J., Chen, F., van Dorpe, J. \& Nitsch, R. M. Formation of neurofibrillary tangles in P301I tau transgenic mice induced by Abeta 42 fibrils. Science 293, 1491-1495 (2001). 
33. Oddo, S., Caccamo, A., Kitazawa, M., Tseng, B. P. \& LaFerla, F. M. Amyloid deposition precedes tangle formation in a triple transgenic model of Alzheimer's disease. Neurobiol. Aging 24, 1063-1070 (2003).

34. Dumont, M. et al. Behavioral deficit, oxidative stress, and mitochondrial dysfunction precede tau pathology in P301S transgenic mice. FASEB J. 25, 4063-4072 (2011).

35. Elipenahli, C. et al. Behavioral improvement after chronic administration of coenzyme Q10 in P301S transgenic mice. J. Alzheimers Dis. 28, 173-182 (2012).

36. Zhao, Y. \& Zhao, B. Oxidative stress and the pathogenesis of Alzheimer's disease. Oxid. Med. Cell Longev. 2013, 316523 (2013).

37. Cai, S. Q. \& Sesti, F. Oxidation of a potassium channel causes progressive sensory function loss during aging. Nat. Neurosci. 12, 611-617 (2009).

38. Sesti, F. Oxidation of $\mathrm{K}(+)$ channels in aging and neurodegeneration. Aging Dis. 7, 130-135 (2016).

39. Rehman, S. U. et al. Inhibition of c-Jun N-terminal kinase protects against brain damage and improves learning and memory after traumatic brain injury in adult mice. Cereb. Cortex 28, 1-19 (2017).

40. Lagas, J. S. et al. Brain accumulation of dasatinib is restricted by P-glycoprotein (ABCB1) and breast cancer resistance protein (ABCG2) and can be enhanced by elacridar treatment. Clin. Cancer Res. 15, 2344-2351 (2009).

41. Agarwal, S. et al. Active efflux of dasatinib from the brain limits efficacy against murine glioblastoma: broad implications for the clinical use of molecularly targeted agents. Mol. Cancer Ther. 11, 2183-2192 (2012).
42. Chen, Y. et al. P-glycoprotein and breast cancer resistance protein influence brain distribution of dasatinib. J. Pharmacol. Exp. Ther. 330, 956-963 (2009).

43. Mittapalli, R. K. et al. ABCG2 and ABCB1 limit the efficacy of dasatinib in a PDGF-B-driven brainstem glioma model. Mol. Cancer Ther. 15, 819-829 (2016).

44. Vogelgesang, S. et al. Deposition of Alzheimer's beta-amyloid is inversely correlated with P-glycoprotein expression in the brains of elderly nondemented humans. Pharmacogenetics 12, 535-541 (2002).

45. van Assema, D. M. et al. P-glycoprotein function at the blood-brain barrier: effects of age and gender. Mol. Imaging Biol. 14, 771-776 (2012).

46. van Assema, D. M. et al. Blood-brain barrier P-glycoprotein function in healthy subjects and Alzheimer's disease patients: effect of polymorphisms in the ABCB1 gene. EJNMMI Res. 2, 57 (2012).

47. Cirrito, J. R. et al. P-glycoprotein deficiency at the blood-brain barrier increases amyloid-beta deposition in an Alzheimer disease mouse model. J. Clin. Invest 115, 3285-3290 (2005).

48. Aigner, L. et al. Overexpression of the neural growth-associated protein GAP43 induces nerve sprouting in the adult nervous system of transgenic mice. Cell 83, 269-278 (1995).

49. Cotella, D. et al. An evolutionarily conserved mode of modulation of Shaw-like $\mathrm{K}(+)$ channels. FASEB J. 27, 1381-1393 (2013).

50. Longhi, L. et al. Temporal window of vulnerability to repetitive experimental concussive brain injury. Neurosurgery 56, 364-374 (2005). 